\title{
Why I love metabolites in safety testing (MIST): a light-hearted historical perspective
}

"Old science was dusted off, venerable Professors were reinvigorated, new science was invented, new companies touted the ability to define and understand metabolites with their new technologies..."

I love metabolites in safety testing (MIST): at least, now I do. In addition to the real benefits, look at all of the fun we had with it. Aside from providing many of our prestigious scientific journals with some of the most innovative manuscript titles since the invention of Viagra ('Seeing Through the MIST', 'Which Metabolites Have We MIST', 'Clearing the MIST' and 'Gorillas in the MIST', etc.), it has been a roller coaster of activity. In this editorial, I will provide an extremely light-hearted personal interpretation of the historical course of MIST, having a little fun with us in the process. However, on a more serious note, I actually love MIST because the complexities and the processes associated with it have significantly advanced both the applied science of metabolites in drug development and the science of interactions between pharmaceutical company colleagues and regulatory agencies.

\section{The triathlon begins}

Metabolites in safety testing has not been a single event but a marathon, or perhaps more accurately, a triathlon. The starting point of this triathlon was an open workshop between regulators and pharmaceutical companies in 2000 and, most importantly, the initial - now so famous I do not even have to name it - scientific metabolism paper published in 2002. It was painstakingly put together by pharma's 'best and brightest' and reviewed in great detail by possibly our 'slightly less-best and brightest' scientists (me included) before the manuscript was sent on its merry way. We all thought we were being very sensitive and extremely scientific. The proposal was the first to really try to bring more standardization across the unstructured field of metabolites (and who does not want that?). We assumed that the authors would get the accolades they fully deserved for taking that courageous step to rationally solve one of the most poorly defined areas in drug development. But then a funny thing happened. We heard a really big 'wait a minute' from the Washington/Maryland area, essentially saying 'nice try on the manuscript but we have some issues'. It seemed that while regulators agreed that there was complexity around metabolites, possibly our unilaterally designed and scripted approach had its flaws. They may also have justifiably felt that the strong recommendations, eloquently and logically presented in a prestigious journal, by equally prestigious (albeit well-meaning) authors, would essentially become a default guidance, pushing the regulators into a position they did not feel was warranted by their experiences.

"When the official guidance was issued in 2008, it contained some new recommendations not previously emphasized in our scientific discussions..."

This reaction kicked off the second round (i.e., the swimming leg of the triathlon). However, it was a little more interesting than a normal swimming leg. The US FDA published their draft metabolite guidance in June 2006 and it was a well-thought-out document but with some critical topics left open. The pharmaceutical industry and the academic community all rose to the occasion, as now we had another cause to rally around, study and publish on. Old science was dusted off, venerable Professors were reinvigorated, new science was invented, new companies touted the ability to define and understand metabolites with their new technologies; all remarkable stuff! We (pharma) held our own meetings to figure how to fix the problem 'they' caused; regulators held meetings

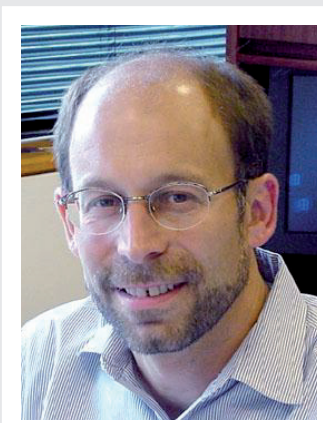

Alfred Tonelli

Global Preclinical Develpment and External Research and Early Development, Johnson and Johnson, Pharmaceutical Researach and Development, Raritan, NJ, USA

E-mail: atonelli@its.jnj.com

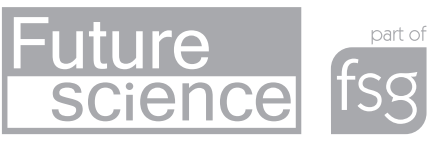


to figure out how to fix the problem 'we' caused. Ultimately, we held a jointly sponsored meeting in Washington DC, USA, with FDA-PhRMADruSafe and Drug Metabolism to present unbiased data and unbiased case studies. In truth, from both the scientific and interactive perspectives, it was one of the best FDA-pharma meetings I have attended. We had real case studies from the FDA; they showed us that the issues were not so clear or so easy, we had real data from pharmaceutical scientists; showing that of course, the path was clear and easy. At the end of the meeting, everyone was exhausted, declared victory and went back to our laboratories. And we all waited and sat, mostly satisfied.

Around December 2007, we were told to get our bicycles ready for MIST-athlon leg number 3: the FDA was about to issue the final guidance. 'Finally', we thought with some fear; what about all of our comments? Were they accepted, rejected or was there something new? However, with our brilliant arguments and science presented to the agency scientists over triathlon legs 1 and 2, we were confident that they too had seen the light and we would get what we justly deserved. But then another funny thing happened. When the official guidance was issued in 2008, it contained some new recommendations not previously emphasized in our scientific discussions. At first glance, some of these appeared extremely constrictive. We wondered how significant changes, such as percentages, total drug-related material and timing on when information was needed, could be made even more conservative without more discussion. But the bottom line was that we now had the official guidance, the horse was out of the barn and we had to deal with it.

\section{"...we also found a lot of very positive things in the Guidance that amplified some of our major points ... the US FDA had ... left room for case-by-case science-based assessments..."}

As good industry representatives, we did what we do well: we huddled, righteously complained and thought about paths forward. Being innovative leaders from many pharma companies, as expected, we had some unique suggestions for moving forward. We rejected multiple thoughts about staging some sort of reenactment of the Boston Tea Party against the FDA in the Potomac. None of us really wanted to bring our boarding boats from New Jersey to Washington. With no really good recommendations forthcoming, we decided we should actually fully study the guidance. We found it quite interesting. We did find things that we still did not like and that were alarming, but we also found a lot of very positive things in the guidance that amplified some of our major points. We also saw that the FDA had, in many areas, not overscripted the guidance but left room for case-by-case science-based assessments. Our thoughts shifted from active reversal to how to continue the learning process for us and the FDA so that this applied science continued to evolve.

\section{"Pharmaceutical companies and global regulatory agencies are still learning and looking for scientific-based proposals to optimize the guidance..."}

"Life is a journey and not a destination", Ralph Waldo Emerson and Steven Tyler: in spite of the fun I had above with this loose history, in truth, I think the events that drove MIST are all the things that make our industry, our scientists and our regulators so effective. The scientists who painstakingly developed the original drug metabolites in safety testing manuscript did so to address a significant gap in our knowledge and to bring a level of consistency to one of the murkiest areas of the development process. It was an extremely well-thought-out document, reflecting the cumulative experience of pharmaceutical scientists with multiple successful filings and very strong links to drug metabolism, bioanalytical, biochemistry, toxicology and regulatory assessments. The principles in the manuscript significantly elevated the practical applications of drug metabolism. However, we must recognize that the regulatory side of the aisle wears different glasses and faces different constraints. The sheer volume of drug applications submitted to our agencies means that there exist large varieties in drug targets, characteristics and resources available from the submitting companies and, ultimately, in the quality of the science being submitted. The one thing that must be consistent is that the applied regulatory standards must ensure safety across a wide range of targets, often resulting in tighter controls than proposed by large pharma.

The second area where we gained as an industry was in developing and harnessing 
new technologies to suit our needs. In the past 10 years, the explosion in instrumentation hardware and software has provided us with tools that are so good they sometimes outstrip our needs. We risk digging holes a mile deep, because we have some really good shovels, when the answers are just below the surface. With MIST in the headlines, scientists felt a lot of pressure to push and exploit the technology to fit their needs and were therefore very focused on getting specific answers around metabolites. With somewhat clear statements in the official guidance regarding conjugated metabolites and low-level metabolites, species requirements and other considerations, the industry was able to focus in on the more important metabolite targets.

Perhaps the greatest positive impact of the MIST storm was the process. MIST was a focal point, where everyone in the preclinical area understood that, in spite of our technical wizardry, there were a lot of gray areas on how best to apply it. The MIST triathalons forced workshops, working groups and cross-pharma interactions in a very open and learning environment. Best practices started evolving from these discussions and were rapidly published or reviewed at scientific meetings. As an industry we became much more efficient in developing and sharing noncompetitive technologies, ultimately increasing speed and reducing costs.

Interactions with regulatory agencies grew in general during this period, but especially in the area of drug metabolism, which, because it is somewhere between clinical and preclinical, can be neglected. Boundaries were clearly present but more objective discussions in neutral environments evolved much more into active learning sessions compared with original sessions, which were often entitled industry position versus FDA position versus academic position.

With all of the progress, MIST is not finished, the dialog is still open. Pharmaceutical companies and global regulatory agencies are still learning and looking for scientific-based proposals to optimize the guidance. Global regulatory agencies are taking the next step and incorporating our follow-on discussions into the International Conference on Harmonization of Technical Requirements for Registration of Pharmaceuticals for Human Use guidance and local practices. We are still pushing technology, and ourselves, to refine the real question and the best way to answer it, as the goal for all of us is still to bring new effective medicines to people as safely and rapidly as possible.

Financial \& competing interests disclosure

Alfred Tonelli is an employee of Johnson and Johnson Pharmaceutical Research and Development. L.L.C., USA. The commentary offered represents purely personal reflections by the author. The author has no other relevant affiliations or financial involvement with any organization or entity with a financial interest in or financial conflict with the subject matter or materials discussed in the manuscript. This includes employment, consultancies, honoraria, stock ownership or options, expert testimony, grants or patents received or pending, or royalties.

No writing assistance was utilized in the production of this manuscript. 